\title{
A dor e a perda dentária: representações sociais do cuidado à saúde bucal
}

\author{
The toothache and toothloss: \\ social representation of oral care
}

Aurigena Antunes Araújo Ferreira 1

Grasiela Piuvezam 2

Carlos Wagner de Araújo Werner 3

Maria do Socorro Costa Feitosa Alves 2

\footnotetext{
1 Departamento de Biofísica e Farmacologia, UFRN.

Av. Sen. Salgado Filho, s/n.

Campus Universitário,

Lagoa Nova, 59072-920,

Natal RN.

aurigenantunes@ig.com.br

2 Programa de Pós-

Graduação em Ciências

da Saúde, UFRN.

3 Faculdade de Odontologia

da Universidade Metodista

de Piracicaba.
}

Abstract The objective of this study was identify the social representations of the oral care on daily activities to understand how the common sense works with the oral health, we realized the research with Brazilian northeast people. Thirty residents of Cidade da Esperança District, Natal, $R N$, Brazil were analyzed. The data was obtained from a semi-structured interview. The interviews were analyzed by Technique of Content Analysis (Bardin 1 ) as well as by Alceste 4.5 software. The categories were Toothache and Tooth Loss. The Toothache shows how the public health service is restrict. The Tooth Loss shows how the dentistry practice resolved the toothache, and how the prosthetics is limited by the social condition. The Social Representations of the oral care on daily activities show the negative consequences of the dentistry practice and the necessity of the new paradigm to the organization of the public health service taking into consideration the common sense about the oral care on daily activities.

Resumo O objetivo do estudo foi apreender as representações sociais do cuidado à saúde bucal, buscando a compreensão desse saber construído na prática vivida por uma população de baixa renda do Nordeste brasileiro. Foram investigados 30 individuos moradores do bairro de Cidade da Esperança, Natal (RN) Brasil. A coleta de dados, realizada através de uma entrevista semi-estruturada, foi averiguada pela Análise de Conteúdo Temática (Bardin 1 ) e pelo software Alceste 4,5. A análise dos dados possibilitou a construção das categorias: Experiência de Dor e Perda Dentária. Se por um lado a Experiência de Dor indica o acesso restrito aos serviços odontológicos, por outro a Perda Dentária denuncia a existência de uma prática mutiladora imposta pelos serviços de saúde pública para solucionar a dor. Nesta categoria está presente ainda a prótese dentária, cujo desejo tem a sua possibilidade limitada pelas condições econômicas. As representações sociais do cuidado à saúde bucal salientam as conseqüências negativas da prática odontológica vigente e aponta para a necessidade de reestruturação dos serviços, levando em conta a percepção da população em relação ao cuidado à saúde bucal. Palavras-chave Representação Social, Saúde bucal, Perda dentária, Dor de dente 


\section{Introdução}

As ações de cuidado à saúde bucal, historicamente, têm sido estruturadas como uma prática caracterizada pelo curativismo. Entretanto, nas últimas décadas tem se reorganizado em torno de ações direcionadas à promoção à saúde bucal, tanto em países desenvolvidos quanto em países emergentes, alcançando ganhos reais em saúde coletiva (Moyses $\&$ Watt 2 ).

No Brasil, os primeiros dados a respeito da condição de saúde bucal foram apresentados no levantamento epidemiológico realizado em 1986 (Brasil3), que retratou o resultado de uma prática odontológica caracterizada por extrações em massa, incrementando as necessidades de reabilitação com a prótese dentária (Pinto4). Esta situação se devia a uma complexidade de fatores, sendo os mesmos sociais, econômicos e biológicos, com forte influência do modelo curativomutilador oferecido pelos serviços odontológicos, que se caracterizaram por uma prática excludente e assistencialista, voltada para os que podiam financiar os serviços (Mendes 5 ).

O Sistema Único de Saúde (SUS), uma nova formulação política e organizacional para o reordenamento dos serviços e ações de saúde, regulamentado pela Constituição de $1988^{6}$ e pelas Leis Orgânicas da Saúde 8.080 e 8.142 de 1990, cujos princípios doutrinários são a universalidade, eqüidade e integralidade, tornou-se um desafio diante dos antigos modelos assistenciais, pois teria de enfrentar no país uma dívida social acumulada, contribuindo para se pensar novos desafios ao problema da saúde (Mendes 7 , Szwarcwald et al. 8 , Paim ${ }^{9}$.

Apesar da reconhecida importância da saúde bucal, uma parcela considerável da população brasileira não tem acesso aos serviços de saúde. Esta situação é responsável não só pelos números desanimadores vistos no último levantamento epidemiológico nacional, para algumas localidades (Brasil10), mas por demonstrar que as dores de dente e a Perda Dentária estão presentes no cotidiano dos brasileiros (Piuvezam \& Alves ${ }^{11}$, Ferreira \& Alves ${ }^{12}$, Nations \& Nuto ${ }^{13}$, Barros \& Bertoldi14).

A mutilação dentária resultante da perda dos dentes predispõe um estado de doença, pois assinala mudanças físicas, biológicas e emocionais. Os indivíduos desdentados ou usuários de próteses dentárias sentem-se em desvantagem em relação àqueles portadores de dentes naturais (Mendonça15).
Apesar da expansão recente dos serviços de saúde, em uma região rural do Nordeste brasileiro foi constatada que a qualidade do cuidado à saúde bucal é problemática, pois a Perda Dentária estigmatiza a população pobre, reforçando as desigualdades entre as classes sociais (Nations \& Nuto ${ }^{13}$ ).

A dor de dente é vivenciada como dificuldade enfrentada pelas populações de baixa renda, que não encontram nos serviços de saúde pública meios apropriados para o cuidado à saúde bucal (Ferreira \& Alves ${ }^{12}$ ). Na compreensão de uma dada realidade é necessário identificar a maneira como os sujeitos sociais identificam, explicam e elaboram os acontecimentos. No caso do cuidado à saúde bucal, essa realidade em processo está em consonância com a experiência vivenciada nos serviços de saúde. O produto desse processo de (re)tradução da realidade cotidiana são as representações sociais. $O$ conceito de representação social apresenta-se como elemento central dessa proposta de estudo, que será uma via de acesso para o conhecimento das implicações psicossociais da prática odontológica nos serviços de saúde.

Nesta perspectiva, a presente investigação apoiou-se na Teoria das Representações Sociais, que possibilita ao pesquisador centrar-se nas comunicações e condutas que orientam os grupos sociais (Jodelet16, Moscovici17).

Partindo desses pressupostos, o presente estudo tem o objetivo de apreender as representações sociais do cuidado à saúde bucal buscando a compreensão e explicação desse saber construído no cotidiano de uma população de baixa renda do Nordeste brasileiro.

\section{Metodologia}

O estudo foi realizado na cidade de Natal (RN), no bairro de Cidade da Esperança, situado na Região Administrativa Oeste da cidade, sendo considerada uma das regiões mais pobres da cidade.

Participaram 30 indivíduos de ambos os sexos, com idade entre 18 e 72 anos. A definição do número de indivíduos não se fez com base em amostragem probabilística, sendo o número de sujeitos delimitado pela saturação dos discursos (Bauer \& Aarts 18 ).

O processo de integração dos sujeitos nesta investigação se deu por um período de 40 horas no interior da Unidade Integrada de Saúde de Cidade da Esperança (UISCE). A pesquisa- 
dora colocou-se na ante-sala de espera do consultório odontológico buscando relacionar-se com os sujeitos da forma mais próxima possível. Foi iniciada uma abordagem para apresentar o trabalho aos investigados, acolhendo-se como sujeitos os que espontaneamente se propuseram a participar, sendo procurados aqueles que satisfaziam a determinados critérios externos como: idade igual ou maior que 18 anos, ser morador do bairro e usuário de serviços de saúde bucal aí disponíveis. Os selecionados ratificaram seu consentimento com sua assinatura ou impressão digital numa carta que descrevia o estudo. Além disso, cada sujeito designava um pseudônimo como forma de resguardar a sua identidade pessoal. O presente estudo foi apreciado e aprovado pelo Comitê de Ética em Pesquisa da Universidade Federal do Rio Grande do Norte (UFRN).

A coleta de dados se fez por meio de uma entrevista semi-estruturada com um inquérito a respeito do cuidado à saúde bucal e a caracterização socioeconômica, sendo pesquisadas as variáveis sexo, idade, renda, ocupação e anos de estudo. O tempo de entrevista era livre e ficou situado no intervalo de 30 a $60 \mathrm{mi}$ nutos.

Num primeiro momento, as entrevistas foram analisadas a partir da Análise de Conteúdo Temática (Bardin 1 ). No segundo momento, os dados da entrevista foram processados pelo software Alceste 4,5 (Analyse Lexicale par Contexte d'un Ensemble de Segments de Texte) que realiza a análise léxica de um conjunto de dados textuais (Reinert19). O Alceste agrupa as raízes semânticas definido-as por classes, levando em consideração a função da palavra dentro de um dado contexto. Cada classe é composta de várias Unidades de Contexto Elementares (U.C.E.)

Como reporta Kronberger \& Wagner 20 , o Alceste computa para cada classe uma linha de palavras que são características da mesma em que a força da associação entre cada palavra e sua classe é expressa por um valor de Qui-Quadrado $\left(\chi^{2}\right)$. Quanto maior o valor do $\chi^{2}$, mais importante é a palavra para a construção estatística da classe. A lista de palavras é a fonte básica para interpretação das classes.

\section{Resultados e discussão}

O cuidado à saúde bucal tem merecido importantes considerações no contexto das políticas de saúde, pois a prática mutiladora tem implicações psicossociais que acarretam a manifestação de diferentes comportamentos, como intimidação e acanhamento, devido às mudanças biológicas, físicas e emocionais.

Pensar na representação social do cuidado à saúde bucal denota inúmeras implicações de cunho psicossociais e culturais, aos quais são submetidos os usuários dos serviços de saúde.

Neste estudo, o cuidado à saúde bucal foi realizado com indivíduos que apresentam uma ampla variação nas idades (18 a 72 anos), 26,7\% do sexo masculino e $73,3 \%$ do sexo feminino (Quadro 1). Um dos fatores que poderia contribuir para os diferentes significados em relação ao cuidado à saúde bucal seria a atenção diferenciada para as diferentes faixas de idade e sexo, nos serviços de saúde a que têm acesso. No entanto para o perfil dos sujeitos estudados a UISCE apresenta o Atendimento às Urgências (39,9\% de ações medicamentosas e $29,1 \%$ de exodontias) e o Pronto Atendimento (41,7\% de restaurações e $34,3 \%$ de exodontias), em que predominam atividades curativas e mutiladoras (Ferreira \& Alves ${ }^{12}$ ).

Nessa perspectiva, o espaço social dos serviços odontológicos voltados para os cuidados à saúde bucal pode favorecer um novo olhar, quando tais serviços são explorados a partir das representações sociais, perpassando a esfera normativa. Sob este novo olhar, eles expressam a subjetividade da realidade, em vez de se restringirem às questões técnicas.

Considerando os discursos dos sujeitos, foi possível identificar pela Análise de Conteúdo de Temática as categorias: Perda Dentária e Experiência de Dor, sendo apontadas na percepção do cuidado à saúde bucal. Na tabela 1 estão apresentadas a freqüência das categorias e os seus respectivos percentuais.

No Programa Alceste 4,5 foram identificadas, nas 30 entrevistas, 61 U.C.E (Unidade de Contexto Elementar), sendo 48 U.C.E. $(78,7 \%)$

\begin{tabular}{lcc}
\hline $\begin{array}{l}\text { Tabela } \mathbf{1} \\
\text { Distribuição das categorias da representação } \\
\text { social do cuidado à saúde bucal, Natal, Brasil, }\end{array}$ \\
\begin{tabular}{lcc}
2005. \\
\hline Categorias & $\begin{array}{c}\text { Freqüência } \\
\text { Absoluta }\end{array}$ & $\begin{array}{c}\text { Freqüência } \\
\text { Percentual \% }\end{array}$ \\
\hline & 119 & 70,4 \\
\hline $\begin{array}{l}\text { Perda dentária } \\
\text { Experiência de dor }\end{array}$ & 50 & 29,6 \\
Total & $\mathbf{1 6 9}$ & $\mathbf{1 0 0 , 0}$ \\
\hline
\end{tabular}
\end{tabular}


pertencentes à classe 1 , ou seja, à classe denominada Perda Dentária, e 13 U.C.E (21,3\%) à classe 2, chamada Experiência de Dor.

Na figura 1 são apresentadas as duas classes identificadas pelo Programa Alceste 4,5.

Nesse estudo emergiram conteúdos compartilhados a respeito do cuidado à saúde bucal evidenciando acontecimentos que estão estritamente relacionados, retratando o contexto social e de saúde pública em que os sujeitos estão inseridos.

\section{A experiência de dor}

A dor é um sintoma que causa desordem, mas que ao mesmo tempo suscita uma ordem, pois faz com que os homens se organizem socialmente para enfrentá-la, seja utilizando conhecimentos de ordem científica, ou de ordem simbólica como podem ser identificados através dos discursos dos sujeitos a seguir:

[...] Há muito tive dor de dente, eu coloquei aguardente (cachaça) para parar [...] (Dedicada)

[...] Já tive dor de dente foi horrivel, para parar ensinaram alho machucado e coloquei em ci$m a[\ldots]$ (Noite)
[...] Eu morria de dor de dente, uma vez estava tão doida de dor de dente que eu coloquei creolina. Até o médico brigou [...] (Tolerância)

As representações sociais do cuidado à saúde bucal permitem apreender que em um meio social existem práticas culturais, que em muitos casos se constituem como a única alternativa para atender às dores e sofrimentos. Para os sujeitos desse estudo, a Experiência de Dor foi identificada como categoria em $29,6 \%$ dos discursos.

A secularização da ciência trouxe consigo a necessidade de repensar as suas interpretações que também não podem ser compreendidas de um ponto de vista fixo, não sendo, portanto, nem definitivas, nem únicas ou exclusivas de um modo de pensar sobre a sociedade. No que toca às formas alternativas de cura, tem-se um amplo leque de produções culturais, que escapam ao controle do saber erudito, revelando que a penetração da cultura erudita não recobriu todos os espaços sociais, absorvendo-as ou assimilando-as sob sua lógica de dominação simbólica (Oliveira21).

A Experiência de Dor é um sintoma presente nas classes sociais desfavorecidas, que chegam a esta extremidade devido a uma di-

Figura 1

Perfil das classes do Alceste para os usuários dos serviços de saúde do Bairro de Cidade da Esperança, Natal, RN, 2005.

Representação social do cuidado à saúde bucal

\begin{tabular}{llc} 
& \multicolumn{2}{c}{} \\
\hline Classe 1 & $\mathbf{7 8 , 7 \%}$ \\
\hline \multicolumn{3}{c}{ Perda Dentária } \\
\hline$\chi^{2}$ & Termo & Freqüência \\
\hline 6,38 & Comer & 16 \\
5,23 & Falta & 24 \\
4,47 & Falar & 15 \\
3,78 & Prótese & 15 \\
2,54 & Tenho & 14 \\
2,14 & Pessoa & 07 \\
2,14 & Coisa & 07
\end{tabular}

\begin{tabular}{llc}
\hline Classe 2 & \multicolumn{2}{c}{$\mathbf{2 1 , 3 \%}$} \\
\hline \multicolumn{3}{c}{ Experiência de Dor } \\
\hline$\chi^{2}$ & Termo & Freqüência \\
\hline 20,11 & Ficha & 6 \\
15,26 & Dormir & 5 \\
11,65 & Dor & 5 \\
11,65 & Tratar & 3 \\
7,36 & Precisar & 3 \\
4,86 & Importante & 3 \\
3,87 & Começar & 2 \\
3,87 & Deixar & 2 \\
3,27 & Tempo & 3 \\
3,27 & Trabalhar & 3 \\
2,9 & Extrair & 5 \\
\hline
\end{tabular}


versidade de fatores sociais, dentre eles, a jornada de trabalho. Esta, entre as classes operárias, não permite que o empregado se ausente do serviço para cuidar preventivamente da saúde bucal, mas apenas quando existe a dor, uma vez que a mesma compromete o desempenho do trabalhador (Souza 22 , Martins'23).

O cuidado à saúde bucal não pode estar desarticulado das condições socioeconômicoculturais, pois na sociedade capitalista, marcada por desigualdades sociais, existe uma ideologia de que o corpo humano é feito para produzir, como assim constatou Souza 22 em um estudo realizado com classes populares. Para Boltanski24, os membros das classes populares não prestam voluntariamente atenção ao seu corpo, pois o usam principalmente como instrumento, subordinando-o às funções sociais. Sendo assim, a doença se manifestará brutal- mente porque não se aperceberam dos sinais precursores ou porque se recusaram a percebê-los.

Os sujeitos ora estudados apresentam uma condição social e econômica preocupante, pois, quanto à ocupação, a maior parte dos homens (37,5\%) está desempregada e $54,5 \%$ e as mulheres em sua maioria são donas de casa (Quadro 1). A seguir o discurso de um dos entrevistados: [...] Eu demoro a ir ao dentista porque não posso faltar ao trabalho [...] (Noite).

Outro fator que justifica a Experiência de Dor é o acesso aos serviços odontológicos. O senso comum pode revelar que nesse momento em que vigora a Política Nacional de Saúde Bucal do Sistema Único de Saúde (SUS), o acesso universal garantido permanece no plano constitucional. Na realidade, $\mathrm{o}$ acesso aos serviços odontológicos é baseado num modelo

\begin{tabular}{|c|c|c|c|c|c|}
\hline \multicolumn{6}{|c|}{$\begin{array}{l}\text { Quadro 1 } \\
\text { Caracterização socioeconômica dos sujeitos, Natal, RN, } 2005 .\end{array}$} \\
\hline Pseudônimo & Sexo & Idade & Ocupação & Renda (R\$) & $\begin{array}{l}\text { Anos de } \\
\text { Estudo }\end{array}$ \\
\hline Lua & M & 21 & Estudante & 300,00 & 9 \\
\hline Vida & M & 53 & Vendedor & 180,00 & Analfabeto \\
\hline Sol & $\mathrm{F}$ & 31 & Do lar & 360,00 & 8 \\
\hline Terra & $\mathrm{F}$ & 58 & Vigilante & 305,00 & 1 \\
\hline Estrela & $\mathrm{F}$ & 38 & Do lar & Não sabe & 5 \\
\hline Água & $\mathrm{F}$ & 29 & Comerciante & 800,00 & 11 \\
\hline Tristeza & M & 30 & Desempregado & 180,00 & 10 \\
\hline Fogo & M & 18 & Estudante & Não sabe & 10 \\
\hline Felicidade & $\mathrm{F}$ & 36 & Do lar & 180,00 & 4 \\
\hline Luz & $\mathrm{F}$ & 43 & Do lar & 200,00 & 6 \\
\hline Calor & $\mathrm{F}$ & 32 & Do lar & 180,00 & 4 \\
\hline Saudade & M & 42 & Desempregado & Sem renda & 11 \\
\hline Emoção & $\mathrm{F}$ & 43 & Do lar & 95,00 & 5 \\
\hline Brilho & $\mathrm{F}$ & 28 & Cozinheira & 220,00 & 10 \\
\hline Experiência & M & 61 & Carregador & 200,00 & Analfabeto \\
\hline $\mathrm{Paz}$ & $\mathrm{F}$ & 72 & Pensionista & 170,00 & 4 \\
\hline Esperança & $\mathrm{F}$ & 30 & Do lar & Sem renda & 5 \\
\hline Verdade & M & 23 & Desempregado & Não sabe & 11 \\
\hline Amizade & $\mathrm{F}$ & 30 & Do lar & Não sabe & 4 \\
\hline Tolerância & $\mathrm{F}$ & 40 & Desempregada & 450,00 & 11 \\
\hline União & $\mathrm{F}$ & 31 & Do lar & 200,00 & 11 \\
\hline Fortaleza & $\mathrm{F}$ & 45 & Autônoma & $1.400,00$ & 11 \\
\hline Dedicada & $\mathrm{F}$ & 24 & Do lar & Não sabe & 8 \\
\hline Humilde & $\mathrm{F}$ & 51 & Desempregada & 180,00 & 3 \\
\hline Bondade & $\mathrm{F}$ & 29 & Do lar & 480,00 & 11 \\
\hline Alegria & M & 66 & Aposentado & 390,00 & 1 \\
\hline Noite & $\mathrm{F}$ & 33 & Auxiliar de costura & 180,00 & 3 \\
\hline Solidão & $\mathrm{F}$ & 49 & Pensionista & 370,00 & 4 \\
\hline Dia & $\mathrm{F}$ & 37 & Feirante & 400,00 & 1 \\
\hline Sonhadora & $\mathrm{F}$ & 27 & Do lar & $1.000,00$ & 11 \\
\hline
\end{tabular}


tradicional de retirada de fichas para o tratamento das doenças, como revela o discurso abaixo: [...] Quando cuido da boca, dependendo do lugar, no interior é mais difícil, a Prefeitura é desorganizada, a ficha é para os favorecidos politicos. A rotina daqui é muito ruim, para quem é preguiçoso. Tem que vir de 4 horas, 3 horas. (Verdade)

A perspectiva traçada sublinha a importância da Teoria das Representações Sociais na prática odontológica, na medida em que permite verificar como os indivíduos constroem, orientam-se e são guiados diante dos acontecimentos com que se deparam (Valsiner25, 28, Doise ${ }^{26}$, Branchs 27 ).

Trata-se então de se pensar que a dor há de ser resolvida de alguma forma. A odontologia, possuidora de inúmeras tecnologias, ainda convive com recursos que não trazem benefícios aos indivíduos como é o caso da Perda Dentária. Mas essa prática mutiladora rudimentar, que marcou os primórdios da profissão, delata as extremidades de uma prática de saúde odontológica que representa e reproduz as desigualdades sociais, indicando, através dos discursos, que a mutilação dentária/perda dentária ainda se constitui numa prática contemporânea e cotidiana, a ser discutida a seguir.

\section{Perda dentária}

A Perda Dentária é mais um agravante a comprometer uma condição biológica, a mastigação, que se encontra limitada socialmente pela baixa renda, visto que $76,7 \%$ ganham até 3 salários mínimos (Quadro 1). A Perda Dentária aparece como categoria emergente no discurso dos sujeitos em 70,4\%, de forma que é possível verificar que a mesma subsidia limitações quando da mastigação dos alimentos a ser vista no discurso a seguir:

[...] Os dentes faltando é ruim para comer [...] (Experiência)

[...] Fazem falta os dentes, pois para comer tem que jogar a comida para o lado onde estão os dentes, a não ser que coma papa [...] (Terra)

As dificuldades enfrentadas pelas limitações impostas podem ser superadas, de acordo com os sujeitos investigados pela colocação da prótese dentária, que teria a finalidade de substituir parte do corpo que outrora fora perdido. Isto permite que seja criada uma expectativa nos indivíduos, como se verifica na fala a seguir:

Eu vou ficar muito animada com a prótese! Eu acho que vai ser o dia mais feliz da minha vi- da, pois é bom para falar, comer e sorrir. Eu adoro sorrir, com vergonha às vezes eu até deixo de sorrir, um sorriso alegre, sadio [...] (Emoção)

Ao mesmo tempo, em que é atribuída como solução para a Perda Dentária, a prótese, segundo os que dela necessitam, tem acesso limitado, porque não está disponível na saúde pública. Os serviços públicos odontológicos acabam por caracterizar-se como mutiladores, pois comprometem a imagem dos indivíduos. No trabalho de Mendonça15, o desdentamento foi apontado como saída para o fracasso do tratamento conservador anteriormente realizado.

Com isto, percebe-se que as dificuldades econômicas podem fazer com que a prótese se configure nos sonhos e imaginário, sem contudo fazer com que tenha condições de apresentar a imagem dentada:

[...] Gostaria de colocar uma peça, mas não tenho condições econômicas, nem é tanto caro, mas sem trabalhar!!! Os dentes são importantes em tudo, não pode comparar com uma prótese [...] (União)

[...] Eu extraí meus dentes, mas eu falo bem [...] (risos) mas não tenho condições de colocar a prótese [...] (Esperança)

[...] Não coloquei a prótese porque não tenho condições ]...] (Felicidade)

Nesse quadro, vale a pena lembrar que a extração dentária e a substituição por meios artificiais mostram-se como avanço técnico num determinado momento. Hoje representam o resquício de uma concepção e de uma prática de saúde bucal. Eliminando um sintoma da doença, pela extração, restaura a condição de normalidade (não sentir dor), mas instaura a anormalidade (não ter dentes), que pode ser reconduzida à sua normalidade por meio de uma prótese dentária. Assim, o que é "anormal" passa a ser aceito como "normal", social e mesmo profissionalmente (Iyda 29 ).

A despeito dos avanços obtidos no plano legal, a realidade dos serviços oferecidos à população em muito se distancia da garantia do direito à saúde tão almejado em nosso país. A Experiência de Dor é vivenciada pelos sujeitos, sendo esta sintomatologia que subsidia tanto a busca pelo tratamento, quanto a prática mutiladora nos serviços de saúde pública. A Perda Dentária traz deficiências para a vida cotidiana dos indivíduos, que podem ser superadas pela colocação da prótese dentária, cujo acesso está limitado pelas condições econômicas.

O estudo aponta para a necessidade de estudos de natureza qualitativa com levantamen- 
tos epidemiológicos, uma vez que aproxima a odontologia dos problemas vivenciados pela comunidade, para definição de metas que atendam à população em relação ao cuidado com a boca em vez da habitual intervenção normativa do profissional que determina a necessidade de tratamento baseado somente nos parâmetros da doença.

\section{Colaboradores}

AAA Ferreira elaborou o projeto de pesquisa, coletou os dados, realizou as análises, escreveu e corrigiu o artigo. G Piuvezam realizou a coleta e análise dos dados; CWA Werner participou da redação e correção do artigo; e MSC Feitosa Alves orientou o projeto de pesquisa, analisou os dados, escreveu e corrigiu o artigo.

\section{Referências}

1. Bardin L. Análise de conteúdo. Lisboa: Edições 70; 1977.

2. Moyses ST, Watt R. Promoção de saúde bucal definições. In: YP Buisch, organizador. Promoção de saúde bucal na clínica odontológica. São Paulo: Artes Médicas-APCD-EAP; 2000. p. 1-22.

3. Brasil. Ministério da Saúde. Divisão Nacional de Saúde Bucal. Levantamento epidemiológico em saúde bucal-Brasil, zona urbana, 1986. Brasília: MS; 1988.

4. Pinto VG. Epidemiologia das doenças bucais no Brasil. In: L Krieger, organizador. Promoção de saúde bucal. São Paulo: Artes Médicas; 1988. p. 27-42.

5. Mendes EV. A evolução histórica da prática médica: suas implicações no ensino, na pesquisa e na tecnologia médica. Belo Horizonte PUC-MG; Finep; 1985.

6. Constituição da República Federativa do Brasil. Brasília, DF: Senado, 1988.

7. Mendes EV. O dilema do SUS. Radis: Comunicação em Saúde 2004; 25 set:35.

8. Szwarcwald CL, Viacava F, Vasconcellos MTL, Leal MC, Azevedo LO, Queiroz RSB, et al. O Brasil em números. Radis: Comunicação em Saúde 2004; 23 jul:14-33.

9. Paim JS. Descentralização das ações e serviços de saúde no Brasil e a renovação da proposta saúde para todos. Rio de Janeiro: Instituto de Medicina Social; 1998. (Série: Estudos em Saúde Coletiva, 175).

10. Brasil. Ministério da Saúde. Coordenação Nacional de Saúde Bucal. Projeto SB Brasil 2003: Condições de Saúde Bucal da População Brasileira 2002-2003. Brasília: Ministério da Saúde; 2004.

11. Piuvezam G \& Alves MCSF. Estudo psicossocial das perdas dentárias na terceira idade [dissertação]. Natal (RN): Universidade Federal do Rio Grande do Norte; 2004.

12. Ferreira AAA, Alves MSCF. Representações sociais da necessidade de cuidados bucais: um estudo com habitantes do bairro de Cidade da Esperança-Natal (RN) [dissertação]. Natal (RN): Universidade Federal do Rio Grande do Norte; 2002.

13. Nations M K, Nuto SAS. Tooth worms, poverty tattoos and dental care conflicts in Northeast Brazil. Soc Sci Med 2002; 54:229-4.

14. Barros AJD, Bertoldi AD. Desigualdade na utilização e no acesso a serviços odontológicos: uma avaliação em nível nacional. Rev C S Col 2002; 7(4):709-17.

15. Mendonça T. Mutilação dentária: concepções de trabalhadores rurais sobre a responsabilidade pela perda dentária. Public Health Rep 2001; 17:1545-7.

16. Jodelet D. As representações sociais: um domínio em expansão. In: D Jodelet, organizador. As representações sociais. Rio de Janeiro: Eduerj; 2002. p. 17-44.

17. Moscovici S. La psychanalyse, son image et son public. Paris: PUF; 1976.

18. Bauer MW, Aarts B. A construção do corpus: um princípio para a coleta de dados qualitativos. In: M Bauer \& G Gaskell, organizadores. Pesquisa qualitativa com texto, imagem e som. Petrópolis: Vozes; 2002. p. 39-63.

19. Reinert M. Alceste: une méthodologie d'analyse des données textuelles et une aplication. Bull Methodol Sociol 1990; 28:24-54 
20. Kronberger N, Wagner W. Palavras-chave em contexto: análise estatística de textos. In: M Bauer \& $\mathrm{G}$ Gaskell, organizadores. Pesquisa qualitativa com texto, imagem e som. Petrópolis: Vozes, 2002. p. 416-41.

21. Oliveira ER. Representações sociais sobre doenças: os mitos da ciência e os cientistas da magia. In: C Botazzo, SFT Freitas, organizadores. Ciências sociais e saúde bucal: questões e perspectivas. São Paulo: Unesp; 1998. p. 43-85.

22. Souza L. A saúde e a doença no dia-a-dia do povo. Cadernos do Ceas 1982; 77:18-29.

23. Martins EM. Construindo o valor saúde bucal. Rev Ação Col 1999; 2:5-9.

24. Boltanski L. As classes sociais e o corpo. Rio de Janeiro: Graal; 1984.
25. Valsiner J. Beyond social representations: a theory of enablement. Papers on Social Representations 2003; 12:7.1-7.16.

26. Doise W 2001. Atitudes e representações sociais. In: D Jodelet, organizador. Representações sociais. Rio de Janeiro: Uerj; 2001. p. 187-204.

27. Branchs MA 2000. Aproximaciones procesuales y estructurales al estudio de las representationes sociales. Papers on Social Representations 2000; 9:3.1-3.15.

28. Doise W. Da psicologia social à psicologia societal. Psicologia: Teoria e Pesquisa 2002; 18(1):27-35.

29. Iyda M. Saúde bucal: uma prática social. In: C Botazzo, SFT Freitas, organizadores. Ciências sociais e saúde bucal: questões e perspectivas. São Paulo: Unesp; 1998. p. 127-40.

Artigo apresentado em 10/10/2004

Aprovado em 22/03/2005

Versão final apresentada em 7/04/2005 